\title{
ON THE GEOMETRY OF ALGEBRAIC CURVES HAVING MANY REAL COMPONENTS
}

\section{J. HUISMAN}

\begin{abstract}
We show that there is a large class of nonspecial effective divisors of relatively small degree on real algebraic curves having many real components i.e. on $M$-curves. We apply to

1. complete linear systems on $M$-curves containing divisors with entirely real support, and

2. morphisms of $M$-curves into $\mathbb{P}^{1}$.
\end{abstract}

\section{Introduction}

One of the subjects in real algebraic geometry that enjoys considerable attention is the study of the topology of real algebraic plane curves. This study is part of Hilbert's 16th problem and, ever since, quite some progress has been made (see [1] and its references on the subject). Particular interest has been devoted to the case of plane curves having many real components.

Concerning the study of real algebraic plane curves, it is natural to study separately the real algebraic curve and its embedding into the projective plane. Since such an embedding is completely determined by its linear system, the first part of Hilbert's 16th problem may be interpreted as the problem of studying the topology of linear systems on real algebraic curves.

In this paper, we study linear systems on real algebraic curves that have many real components i.e. on $M$-curves. We find a large class of nonspecial effective divisors of relatively small degree on such curves. We apply to

2000 Mathematics Subject Classification: 14P99, 14H45, 14C20.

Servicio de Publicaciones. Universidad Complutense. Madrid, 2001 
1. complete linear systems on $M$-curves containing divisors with entirely real support, and

2. morphisms of $M$-curves into $\mathbb{P}^{1}$.

In particular, we answer for $M$-curves a question of C. Scheiderer.

Convention and notation. A real algebraic curve is a geometrically integral nonsingular proper scheme over $\mathbb{R}$ of dimension 1 . The projective line $\mathbb{P}_{\mathbb{R}}^{1}$ over $\mathbb{R}$ is simply denoted by $\mathbb{P}^{1}$.

\section{Nonspecial effective divisors on $M$-curves}

Let $C$ be a real algebraic curve. A real component of $C$ is a connected component of the set $C(\mathbb{R})$ of real points of $C$. Let $f$ be a nonconstant rational function on $C$. Let $P$ be a closed point of $C$ at which $f$ has a pole. We say that the pole $P$ of $f$ is real if its residue field $k(P)$ is equal to $\mathbb{R}$.

The following statement is the key lemma of the paper. Its proof is so elementary that one may suspect that the statement is well known. Nevertheless, I was not able to find a reference in the literature.

Lemma 2.1. Let $C$ be a real algebraic curve. Let $f$ be a nonconstant rational function on $C$ having only real poles. Suppose that every real component of $C$ contains at most one pole of $f$, counted with multiplicity. Then, every real component of $C$ contains exactly one pole of $f$.

Proof. Let $d$ be the degree of $f$ considered as a morphism from $C$ into $\mathbb{P}^{1}$, i.e. $d=\operatorname{deg}\left(f^{\star} \infty\right)$. Since all the poles of $f$ are real and since each real component of $C$ contains at most one pole of $f$, there are exactly $d$ real components $X_{1}, \ldots, X_{d}$ of $C$ at which $f$ has a pole. The restriction of $f$ to a real component $X_{i}$ is a continuous map from $X_{i}$ into $\mathbb{P}^{1}(\mathbb{R})$ of degree $1(\bmod 2)$ (see $[6]$ for definition and properties of the topological degree). In particular, $f$ maps $X_{i}$ onto $\mathbb{P}^{1}(\mathbb{R})$ for $i=1, \ldots, d$. Since $d=\operatorname{deg}(f)$,

$$
f^{-1}(x) \subseteq \bigcup_{i=1}^{d} X_{i}
$$


for all $x \in \mathbb{P}^{1}(\mathbb{R})$. Hence,

$$
C(\mathbb{R}) \subseteq f^{-1}\left(\mathbb{P}^{1}(\mathbb{R})\right) \subseteq \bigcup_{i=1}^{d} X_{i}
$$

Therefore, for each real component $X$ of $C$ there is $i \in\{1, \ldots, d\}$ such that $X=X_{i}$. In particular, every real component of $C$ contains exactly one pole of $f$.

The following consequence is merely a reformulation of Lemma 2.1:

Corollary 2.2. Let $C$ be a real algebraic curve. Let $s$ be the number of real components of $C$. Let $k<s$ be a natural integer and let $P_{1}, \ldots, P_{k}$ be real points of $C$ such that no two of them belong to the same real component of $C$. Let $D$ be the divisor $\sum_{i=1}^{k} P_{i}$. Then, $h^{0}(D)=1$.

Lemma 2.1, or rather its above corollary, provides yet another proof of Harnack's Inequality for real algebraic curves [4]:

Corollary 2.3. (Harnack's Inequality). Let $C$ be a real algebraic curve. Let $g$ be the genus of $C$ and let $s$ be the number of real components of $C$. Then, $s \leq g+1$.

Proof. Suppose that $s>g+1$. Then there is an effective divisor $D$ on $C$ of degree $k=g+1$ as in Corollary 2.2. By Riemann's Inequality, $h^{0}(D) \geq \operatorname{deg}(D)-g+1=2$ which contradicts the conclusion of Corollary 2.2 .

A real algebraic curve $C$ of genus $g$ is said to have many real components if the number of real components of $C$ is equal to $g+1$; for short, $C$ is called an $M$-curve. One easily convinces oneself of the existence of such curves in any genus. In fact, there are many $M$-curves of given genus: the moduli space of $M$-curves of genus $g$ is a connected semianalytic variety of dimension $3 g-3$, if $g>1[8,5]$.

Recall that a divisor $D$ on an algebraic curve $C$ is said to be nonspecial if $h^{0}(D)=\operatorname{deg}(D)-g+1$, where $g$ is the genus of $C$. By 
Riemann-Roch, $D$ is nonspecial if and only if $h^{0}(K-D)=0$, where $K$ is a canonical divisor on $C$. It follows that $D$ is nonspecial if $D^{\prime}$ is nonspecial and $D \geq D^{\prime}$. Recall also that divisors of degree at least $2 g-1$ are nonspecial.

Using Corollary 2.2, one gets a large class of nonspecial effective divisors of relatively small degree on $M$-curves:

Theorem 2.4. Let $C$ be an $M$-curve of genus $g$. Let $D$ be an effective divisor on $C$ such that $\sup (D) \cap X \neq \emptyset$ for at least $g$ real components $X$ of $C$. Then, $D$ is nonspecial.

Proof. Choose $g$ points $P_{1}, \ldots, P_{g} \in \operatorname{Supp}(D) \cap C(\mathbb{R})$ such that no two of them belong to the same real component of $C$. Put $D^{\prime}=\sum P_{i}$. By Corollary $2.2, h^{0}\left(D^{\prime}\right)=1=\operatorname{deg}\left(D^{\prime}\right)-g+1$. Hence, $D^{\prime}$ is nonspecial. Since $D \geq D^{\prime}, D$ is nonspecial as well.

It is well known that a generic effective divisor of degree at least $g$ is nonspecial. The point of Theorem 2.4 is that it states that all divisors satisfying the explicit conditions of the statement are nonspecial.

In the following sections we discuss some applications of Theorem 2.4 .

\section{$3 \quad$ Morphism into $\mathbb{P}^{1}$}

In this section we use Theorem 2.4 in order to study morphisms of $M$ curves into $\mathbb{P}^{1}$. We start of with the following consequence of Theorem 2.4:

Corollary 3.1. Let $C$ be an $M$-curve and let $g$ be its genus. Let $D$ be an effective divisor on $C$ such that $\operatorname{supp}(D) \cap X \neq \emptyset$ for all real components $X$ of $C$. Then, the linear system $|D|$ is base point-free.

Let $C$ be a real algebraic curve and let $\operatorname{Div}(C)$ denote the group of divisors on $C$. Let $X$ be a real component of $C$ and let $\operatorname{res}_{X}: \operatorname{Div}(C) \rightarrow$ $\operatorname{Div}(C)$ be the restriction-to- $X$ morphism. This morphism is defined by letting $\operatorname{res}_{X}(P)=P$ if $P \in X$ and $\operatorname{res}_{X}(P)=0$ if $P \notin X$, for any closed 
point $P$ of $C$. For any divisor $D$ on $C$, we define the degree of $D$ on $X$ to be the natural number $\operatorname{deg}_{X}(D)=\operatorname{deg}\left(\operatorname{res}_{X}(D)\right)$.

Let $C$ be an $M$-curve and let $g$ be its genus. Let $D$ be an effective divisor on $C$ of degree $g+1$ such that $\operatorname{deg}_{X}(D)=1$ for all real components of $C$. According to Theorem 2.4, $h^{0}(D)=2$, i.e., the linear system $|D|$ on $C$ is 1-dimensional. By Corollary 3.1, $|D|$ is base point-free. Let then $f: C \rightarrow \mathbb{P}^{1}$ be the morphism associated to $D$. The following result states some remarkable properties of $f$.

\section{Proposition 3.2.}

1. The morphism $f: C \rightarrow \mathbb{P}^{1}$ is of degree $g+1$.

2. The restriction of $f$ to any real component of $C$ is a homeomorphism onto $\mathbb{P}^{1}(\mathbb{R})$.

3. For any real point $Q$ of $\mathbb{P}^{1}$, the fiber $f^{-1}(Q)$ is contained in $C(\mathbb{R})$.

4. The morphism $f$ is unramified over each real point of $\mathbb{P}^{1}$.

5. The restriction of $f$ to any real component of $C$ is an analytic isomorphism onto $\mathbb{P}^{1}(\mathbb{R})$.

Proof. Since $|D|$ is base point-free, the degree of $f$ is equal to the degree of $D$, i.e., $\operatorname{deg}(f)=g+1$. This proves Statement 1 .

In order to show Statement 2, we show first that the restriction $\left.f\right|_{X}: X \rightarrow \mathbb{P}^{1}(\mathbb{R})$ of $f$ to any real component $X$ of $C$ is surjective. Indeed, since $\operatorname{deg}_{X}(D)=1$, the topological degree of $\left.f\right|_{X}$ is equal to 1 $(\bmod 2)$. Therefore, $\left.f\right|_{X}$ is surjective.

We show simultaneously the injectivity of $\left.f\right|_{X}$ and Statements 3 and 4: Let $Q$ be a real point of $\mathbb{P}^{1}$. Since $\left.f\right|_{X}$ is surjective for any real component $X$ of $C$, the restriction of $f^{\star} Q$ to any real component of $C$ is a nonzero effective divisor. Then

$$
g+1=\operatorname{deg}\left(f^{\star} Q\right) \geq \sum_{X} \operatorname{deg}_{X}\left(f^{\star} Q\right) \geq g+1 .
$$

Hence the two inequalities are, in fact, equalities. The first of these equalities shows that $f^{\star} Q$ has support contained in $C(\mathbb{R})$. In particular, $f^{-1}(Q)$ is contained in $C(\mathbb{R})$, whence Statement 3 . The second of the two 
equalities shows that the degree of $f^{\star} Q$ on each real component of $C$ is equal to 1. It follows that the restriction of $f$ to any real component of $C$ is injective, whence Statement 2. It also follows that each point of the support of $f^{\star} Q$ appears with multiplicity 1 in $f^{\star} Q$, whence Statement 4 .

Statement 5 follows from Statements 2 and 4 .

We conclude this section with a characterization of $M$-curves in terms of morphisms into $\mathbb{P}^{1}$.

Proposition 3.3. Let $C$ be a real algebraic curve and let $g$ be its genus. Then $C$ is an $M$-curve if and only if there is a morphism $f: C \rightarrow \mathbb{P}^{1}$ satisfying the following 3 conditions.

1. The morphism $f$ is of degree $g+1$.

2. The restriction of $f$ to any real component of $C$ is a homeomorphism onto $\mathbb{P}^{1}(\mathbb{R})$.

3. Any closed point $P \in C$ such that $f(P) \in \mathbb{P}^{1}(\mathbb{R})$ is real.

Moreover, in that case, there is an effective divisor $D$ on $C$ of degree $g+1$ with $\operatorname{deg}_{X}(D)=1$ for all real components $X$ of $C$ such that $f$ is the morphism associated to $D$.

Proof. It follows from Proposition 3.2 that the 3 conditions are necessary. In order to show that they are sufficient, assume that $C$ is a real algebraic curve and that $f: C \rightarrow \mathbb{P}^{1}$ is a morphism satisfying Conditions 1,2 and 3. Let $s$ be the number of real components of $C$. Choose a point $Q \in \mathbb{P}^{1}(\mathbb{R})$ such that $f$ is unramified over $Q$. Then, by Conditions 2 and 3 , the divisor $f^{\star} Q$ is of degree $s$. By Condition $1, s=g+1$.

In order to prove the last statement, observe that the effective divisor $D=f^{\star} \infty$ is of degree $g+1$, by Condition 1 , and satisfies $\operatorname{deg}_{X}(D) \geq 1$ for all real components $X$ of $C$, by Condition 2. Since $C$ has $g+1$ real components, $\operatorname{deg}_{X}(D)=1$ for all real components $X$ of $C$. This shows the last statement of the proposition. 


\section{Divisors with real support}

Let $C$ be an $M$-curve and let $g$ be its genus. Let $\operatorname{Pic}(C)$ denote the Picard group of linear equivalence classes of divisors on $C$. Denote by $\mathrm{cl}$ the morphism from $\operatorname{Div}(C)$ into $\operatorname{Pic}(C)$ that associates to a divisor its class. For an integer $d$, denote by $\operatorname{Div}^{d}(C)$ the subset of $\operatorname{Div}(C)$ consisting of divisors of degree $d$ and let $\operatorname{Pic}^{d}(C)$ be the cl-image of $\operatorname{Div}^{d}(C)$. Recall [3, $2]$ that $\operatorname{Pic}^{0}(C)$ is a compact commutative real Lie group of dimension $g$. Its group of connected components is isomorphic to $(\mathbb{Z} / 2 \mathbb{Z})^{g}$. The neutral component of $\operatorname{Pic}^{0}(C)$ is isomorphic to the real Lie group $\left(S^{1}\right)^{g}$. Moreover, $\operatorname{Pic}^{d}(C)$ is a principal homogeneous space under the action of $\operatorname{Pic}^{0}(C)$. In particular, $\operatorname{Pic}^{d}(C)$ is a compact real analytic manifold of dimension $g$ having $2^{g}$ connected components. Each of its connected components is real analytically isomorphic to the real analytic manifold $\left(S^{1}\right)^{g}$.

Theorem 4.1. Let $C$ be an $M$-curve of genus g. Let $X_{1}, \ldots, X_{g}$ be distinct real components of $C$ and let $X=\prod X_{i}$. Define

$$
\sigma: X \longrightarrow \operatorname{Pic}^{g}(C)
$$

by letting $\sigma\left(P_{1}, \ldots, P_{g}\right)$ be the divisor class $\mathrm{cl}\left(\sum P_{i}\right)$. Then, the map $\sigma$ is a real analytic isomorphism onto a connected component of $\mathrm{Pic}^{g}(C)$.

Proof. Let $C^{(g)}$ denote the $g$-fold symmetric product of $C$. Since the real components $X_{1}, \ldots, X_{g}$ are distinct, the natural map from $X$ into $C^{(g)}(\mathbb{R})$ is a real analytic isomorphism from $X$ onto a connected component of $C^{(g)}(\mathbb{R})$. Identify $X$ with this connected component. Then, $\sigma$ extends to a morphism of real algebraic varieties, again denoted by $\sigma$, from $C^{(g)}$ into $\mathrm{Pic}_{C}^{g}$. Here, $\mathrm{Pic}_{C}^{g}$ denotes the degree- $g$ part of the Picard scheme $\mathrm{Pic}_{C}$ of $C$. The real analytic manifold $\operatorname{Pic}^{g}(C)$ is equal to the set of real points of $\mathrm{Pic}_{C}^{g}$. It is well known that $\sigma$ is a birational morphism. In fact, let $U \subseteq C^{(g)}$ be the subset of nonspecial divisors. Then, $U$ and $\sigma(U)$ are open and nonempty, and $\sigma$ is an isomorphism of $U$ onto $\sigma(U)$. By Theorem 2.4, $X$ is entirely contained in $U$. In particular, $X$ is a connected component of $U(\mathbb{R})$. Then, $\sigma(X)$ is connected component of $\sigma(U)(\mathbb{R})$ and the restriction of $\sigma$ to $X$ is a real analytic isomorphism onto the connected component $\sigma(X)$ of $\sigma(U)(\mathbb{R})$. Since $X$ is compact, $\sigma(X)$ 
is compact and is, therefore, also a connected component of $\operatorname{Pic}^{g}(C)$.

Since the proof of Theorem 4.1 does not use the fact that the connected components of $\operatorname{Pic}^{g}(C)$ are real analytically isomorphic to $\left(S^{1}\right)^{g}$, it provides another proof of this fact.

Theorem 4.1 allows to answer for $M$-curves the following problem formulated by Scheiderer [7, §2]: Given a real algebraic curve $C$ of genus $g$ with $C(\mathbb{R}) \neq \emptyset$, determine explicitly an integer $n$ with the following property. For every complete linear system $|D|$ with $\operatorname{deg}(D) \geq n$ there is $D^{\prime} \in|D|$ such that $\operatorname{Supp}\left(D^{\prime}\right)$ consists of real points only. Indeed, if $C$ is an $M$-curve, the following statement claims that one can take $n=2 g-1$ if $g>0$.

Theorem 4.2. Let $C$ be an $M$-curve of genus $g>0$. Let $d$ be a natural integer and let $\operatorname{Div}_{\mathrm{rs}}^{d, \geq 0}(C)$ be the subset of $\operatorname{Div}^{d}(C)$ of effective divisors having entirely real support. If $d \geq 2 g-1$ then

$$
\operatorname{cl}\left(\operatorname{Div}_{\mathrm{rs}}^{d, \geq 0}(C)\right)=\operatorname{Pic}^{d}(C) .
$$

Proof. Denote the real components of $C$ by $X_{0}, \ldots, X_{g}$. It is well known that if $D$ and $D^{\prime}$ are linearly equivalent divisors on $C$ then $\operatorname{deg}_{X}(D) \equiv \operatorname{deg}_{X}\left(D^{\prime}\right) \bmod 2$ for all real components $X$ of $C$ (see [3, Lemma 4.1]). It follows that

$$
\delta(D)=\left(\operatorname{deg}(D), \operatorname{deg}_{X_{0}}(D), \ldots, \operatorname{deg}_{X_{g}}(D)\right)
$$

defines a morphism $\delta$ from $\operatorname{Pic}(C)$ into $\mathbb{Z} \oplus(\mathbb{Z} / 2 \mathbb{Z})^{g+1}$. The kernel of $\delta$ is the neutral component of $\operatorname{Pic}(C)$. Therefore, the image of $\delta$ is the group of connected components of $\operatorname{Pic}(C)$. It is clear that

$$
\operatorname{im}(\delta)=\left\{\left(x, x_{0}, \ldots, x_{g}\right) \mid x \equiv \sum x_{i} \bmod 2\right\} .
$$

We denote by $x, x_{0}, \ldots, x_{g}$ the coordinates on $\mathbb{Z} \oplus(\mathbb{Z} / 2 \mathbb{Z})^{g+1}$. For $i=$ $0, \ldots, g$, let $e_{i} \in \operatorname{im}(\delta)$ be defined by $x\left(e_{i}\right)=g, x_{i}\left(e_{i}\right)=0$, and $x_{j}\left(e_{i}\right)=$ 1 for $j \neq i$, i.e.,

$$
e_{i}=(g, 1,1, \ldots, 1,0,1, \ldots, 1,1)
$$


where the entry 0 is at the $(i+2)$-nd place. By Theorem 4.1, the connected component $\delta^{-1}\left(e_{i}\right)$ is contained in the cl-image of $\operatorname{Div}_{\mathrm{rs}}^{g, \geq 0}(C)$, for $i=0, \ldots, g$.

Now, it clearly suffices to show the statement for $d=2 g-1$. Choose a connected component of $\mathrm{Pic}^{2 g-1}(C)$. Such a connected component is of the form $\delta^{-1}(e)$ for some $e \in \operatorname{im}(\delta)$. One has $x(e)=2 g-1$. We show that there is an effective divisor $D$ on $C$ of degree $g-1$ with real support such that the translation-by-cl $(D)$ map on $\operatorname{Pic}(C)$ maps a connected component of $\operatorname{Pic}(C)$ of the form $\delta^{-1}\left(e_{i}\right)$ onto $\delta^{-1}(e)$. Since $\delta^{-1}\left(e_{i}\right)$ is contained in $\operatorname{cl}\left(\operatorname{Div}_{\mathrm{rs}}^{g, \geq 0}(C)\right)$, it will imply that $\delta^{-1}(e)$ is contained in the cl-image of $\operatorname{Div}_{\mathrm{rs}}^{2 g-1, \geq 0}(C)$.

There are two cases to consider:

First case: all coordinates $x_{i}(e)$ are nonzero. Since $e \in \operatorname{im}(\delta)$, one has $2 g-1 \equiv g+1 \bmod 2$, i.e., $g$ is even. Let $P$ be any real point contained in $X_{0}$. Let $D$ be the divisor $(g-1) P$. Let $\tau$ be the translation-by-cl $(D)$ map on $\operatorname{Pic}(C)$. Since $g-1$ is odd, $\tau\left(\delta^{-1}\left(e_{0}\right)\right)=\delta^{-1}(e)$. Since $D$ is an effective divisor with real support, it follows that $\delta^{-1}(e)$ is contained in the cl-image of $\operatorname{Div}_{\mathrm{rs}}^{2 g-1, \geq 0}(C)$.

Second case: there is an integer $i$ such that $x_{i}(e)=0$. Since $2 g-1$ is odd, there is an integer $j$ such that $x_{j}(e)=1$. For any integer $k$ such that $x_{k}(e) \neq x_{k}\left(e_{i}\right)$, choose a point $P_{k} \in X_{k}$. Let

$$
D^{\prime}=\sum_{x_{k}(e) \neq x_{k}\left(e_{i}\right)} P_{k}
$$

Since $x_{i}(e)=0=x_{i}\left(e_{i}\right)$ and $x_{j}(e)=1=x_{j}\left(e_{i}\right)$, the degree of $D^{\prime}$ is at most $g-1$. Moreover,

$$
\operatorname{deg}\left(D^{\prime}\right) \equiv \sum_{k=0}^{g} x_{k}(e)-x_{k}\left(e_{i}\right) \equiv(2 g-1)-g \equiv g-1 \bmod 2 .
$$

Therefore, there is a nonnegative integer $\ell$ such that $\operatorname{deg}\left(D^{\prime}\right)+2 \ell=$ $g-1$. Choose a point $P_{i} \in X_{i}$ and let $D=D^{\prime}+2 \ell P_{i}$. Let $\tau$ be the translation-by-cl $(D)$ map on $\operatorname{Pic}(C)$. Then, $\tau\left(\delta^{-1}\left(e_{i}\right)\right)=\delta^{-1}(e)$. Since $D$ is an effective divisor with real support, it follows again that $\delta^{-1}(e)$ is contained in the cl-image of $\operatorname{Div}_{\mathrm{rs}}^{2 g-1, \geq 0}(C)$. 
One may wonder whether the sufficient condition $d \geq 2 g-1$ in Theorem 4.2 is also necessary. It is tempting to suspect that it is, i.e., that

$$
\operatorname{cl}\left(\operatorname{Div}_{\mathrm{rs}}^{2 g-2, \geq 0}(C)\right) \varsubsetneqq \operatorname{Pic}^{2 g-2}(C) .
$$

One may also wonder whether the conclusion of Theorem 4.2 holds for any real algebraic curve $C$ of genus $g>0$ with $C(\mathbb{R}) \neq \emptyset$.

\section{References}

[1] Bochnak, J., Coste, M., Roy M-F.: Géométrie algébrique réelle. Ergeb. der Math., 3. Folge, Band 12. Springer Verlag, 1987

[2] Ciliberto, C., Pedrini, C.: Real abelian varieties and real algebraic curves. Lectures in real geometry, F. Broglia (ed.), de Gruyter Exp. Math. 23, $1996,167-256$

[3] Gross, B. H., Harris, J.: Real algebraic curves. Ann. scient. Ec. Norm. Sup. $4^{e}$ série, t. 14 (1981), 157-182

[4] Harnack, A.: Über die Vieltheiligkeit der ebenen algebraischen Curven. Math. Ann. 10 (1876), 189-198

[5] Huisman, J.: Real quotient singularities and nonsingular real algebraic curves in the boundary of the moduli space. Compositio Math. 118 (1999), $43-60$

[6] Milnor, J. W.: Topology from the differentiable viewpoint. Univ. Press Virginia, 1965

[7] Scheiderer, C.: Sums of squares of regular functions on real algebraic varieties. To appear in Trans. Amer. Math. Soc.

[8] Seppälä, M., Silhol, R.: Moduli spaces for real algebraic curves and real abelian varieties. Math. Z. 201 (1989), 151-165

Institut Mathématique de Rennes, Université de Rennes 1, Campus de Beaulieu, 35042 Rennes Cedex, France.

E-mail: huisman@univ-rennes1.fr

Recibido: 22 de Mayo de 2000

Revisado: 4 de Septiembre de 2000 